\title{
Support Vector Machines for Regression and Applications to Software Quality Prediction
}

\author{
Xin $\operatorname{Jin}^{1}$, Zhaodong Liu ${ }^{1}$, Rongfang $\mathrm{Bie}^{1, *}$, Guoxing Zhao ${ }^{2,3}$, and Jixin $\mathrm{Ma}^{3}$ \\ ${ }^{1}$ College of Information Science and Technology, \\ xinjin796@126.com, liuzd661@163.com, rfbie@bnu.edu.cn \\ Beijing Normal University, Beijing 100875, P.R. China \\ ${ }^{2}$ School of Mathematical Sciences, Beijing Normal University, Beijing 100875, P.R. China \\ ${ }^{3}$ School of Computing and Mathematical Science, \\ The University of Greenwich, London SE18 6PF, U.K \\ G. Zhao@gre.ac.uk, \\ j.ma@gre.ac.uk
}

\begin{abstract}
Software metrics are the key tool in software quality management. In this paper, we propose to use support vector machines for regression applied to software metrics to predict software quality. In experiments we compare this method with other regression techniques such as Multivariate Linear Regression, Conjunctive Rule and Locally Weighted Regression. Results on benchmark dataset MIS, using mean absolute error, and correlation coefficient as regression performance measures, indicate that support vector machines regression is a promising technique for software quality prediction. In addition, our investigation of PCA based metrics extraction shows that using the first few Principal Components (PC) we can still get relatively good performance.
\end{abstract}

\section{Introduction}

Software quality management, which is an important aspect of software project development, is an ongoing comparison of the actual quality of a product with its expected quality [16]. Software metrics are the key tool in software quality management. Many researchers have sought to analyze the connection between software metrics and code quality [8][13][14][15][17][23]. The methods they used fall into four mainly categories: association analysis (association rules), clustering analysis ( $\mathrm{k}$ means, fuzzy c-means), classification analysis (decision trees, layered neural networks, Holographic networks, logistic regression, genetic granular classification [12]) and prediction analysis (linear regression).

In this paper we propose to use support vector machines for regression to predict software quality. Support vector machine technique has attracted many researchers in optimization and machine learning areas $[19,22]$. In the case of regression, the objective is to choose a hyperplane with small norm while simultaneously minimizing the sum of the distances from the data points to the hyperplane.

\footnotetext{
* Corresponding author.
} 
The remainder of this paper is organized as follows. In Section 2, we describe the software metrics and benchmark dataset we used. Section 3 presents the support vector regression method. Section 4 describes three comparison algorithms. Section 5 introduces PCA. Section 6 presents the performance measures and the experiment results. Conclusions are covered in Section 7.

\section{Software Metrics}

We investigated the twelve software metrics, as shown in Table 1, which are used in the famous benchmark dataset MIS [15,12]. Simple counting metrics such as the number of lines of source code or Halstead's number of operators and operands describe how many "things" there are in a program. More complex metrics such as McCabe's cyclomatic complexity or Bandwidth attempt to describe the "complexity" of a program, by measuring the number of decisions in a module or the average level of nesting in the module, respectively.

Table 1. Description of the MIS dataset with a detailed characterization of the software metrics [18]

\begin{tabular}{|c|c|}
\hline Metrics & Detailed Description \\
\hline LOC & $\begin{array}{l}\text { Number of lines of code including comments, declarations and the main } \\
\text { body of the code }\end{array}$ \\
\hline$\overline{\mathrm{CL}}$ & Number of lines of code, excluding comments \\
\hline TChar & Number of characters \\
\hline TComm & Number of comments \\
\hline MChar & Number of comment characters \\
\hline DChar & Number of code characters \\
\hline $\mathrm{N}$ & $\begin{array}{l}\text { Halstead's Program Length } \mathrm{N}=\mathrm{N} 1+\mathrm{N} 2, \mathrm{~N} 1 \text { is the total number of } \\
\text { operators, } \mathrm{N} 2 \text { is the total number of operands }\end{array}$ \\
\hline $\mathrm{N}^{\prime}$ & $\begin{array}{l}\text { Halstead's Estimate of Program Length } N^{\prime}=n_{1} \log _{1} n_{1}+n_{2} \log _{2} n_{2}, n_{1} \text { is the } \\
\text { number of unique operators, } n_{2} \text { is the number of unique operands }\end{array}$ \\
\hline NF & Jensen's Estimate of Program Length Metric $\log _{1} n_{1} !+\log _{2} n_{2} !$ \\
\hline $\mathrm{V}(\mathrm{G})$ & $\begin{array}{l}\text { McCabe's Cyclomatic Complexity Metric, where } \mathrm{V}(\mathrm{G})=\mathrm{e}-\mathrm{n}+2 \text {, and e } \\
\text { represents the number of edges in a control graph of } \mathrm{n} \text { nodes }\end{array}$ \\
\hline BW & $\begin{array}{l}\text { Belady's Bandwidth measure } \mathrm{BW}=\left(\Sigma_{\mathrm{i}} \mathrm{L}_{\mathrm{i}}\right) / \mathrm{n}, \mathrm{L}_{\mathrm{i}} \text { represents the number of } \\
\text { nodes at level "i" in a nested control flow graph of n nodes. This measure } \\
\text { indicates the average level of nesting or width of the control flow graph } \\
\text { representation of the program }\end{array}$ \\
\hline Changes & Number of changes \\
\hline
\end{tabular}

In this study, MIS is represented by a subset of the whole MIS data with 390 modules written in Pascal and FORTRAN. These modules consist of approximately 40,000 lines of code. Our goal is to develop a prediction model of software quality in which the number of modifications (changes) is projected on a basis of the values of the 11 software metrics that is used to characterize a software module. We cast the problem in the setting of regression, the explanatory variables are the first eleven soft- 
ware metrics and the dependent variable is the number of changes. Software modules, which have no changes, could be deemed to be fault-free, while software modules with the number of changes being too big, for example, over 10, can be sought as potentially highly faulty modules.

\section{Support Vector Machine Regression (SVR)}

Specifically, the $\varepsilon$-insensitive support vector regression will be used for predicting software quality. In the $\varepsilon$-insensitive support vector regression, our goal is to find a function $f(x)$ that has an $\varepsilon$-deviation from the actually obtained target $y_{i}$ for all training data and at the same time is as flat as possible [10]. Suppose $f(x)$ takes the following form:

$$
f(x)=w x+b \quad w \in X, b \in \mathfrak{R} .
$$

Then, we have to solve the following problem:

Subject to

$$
\begin{aligned}
& \min \frac{1}{2}\|w\|^{2} \\
& y_{i}-w x_{i}-b \leq \varepsilon \\
& w x_{i}+b-y_{i} \leq \varepsilon
\end{aligned}
$$

In the case where the constraints are infeasible, we introduce slack variables. This case is called soft margin formulation, and is described by the following problem.

Subject to

$$
\begin{array}{rl}
\min \frac{1}{2}\|w\|^{2}+C \sum_{i=1}^{l}\left(\xi_{i}+\xi_{i}^{*}\right) & \\
y_{i}-w x_{i}-b & \leq \varepsilon+\xi_{i} \quad \xi_{i} \xi_{i}^{*} \geq 0 \\
w x_{i}+b-y_{i} \leq \varepsilon+\xi_{i}^{*} & C \succ 0
\end{array}
$$

where $C$ determines the trade-off between the flatness of the $f(x)$ and the amount up to which deviations larger than $\varepsilon$ are tolerated. This is called $\varepsilon$-insensitive loss function:

$$
|\xi|_{\varepsilon}=\left\{\begin{array}{cc}
0 & \text { if }|\xi| \leq \varepsilon \\
|\xi|-\varepsilon & \text { if }|\xi| \succ \varepsilon
\end{array}\right\}
$$

By constructing the Lagrangian function, we formulate the dual problem as

$$
\operatorname{Max}-\frac{1}{2} \sum_{i=1}^{l} \sum_{j=1}^{l}\left(\lambda_{i}-\lambda_{i}^{*}\right)\left(\lambda_{j}-\lambda_{j}^{*}\right) x_{i} x_{j}-\varepsilon \sum_{i=1}^{l}\left(\lambda_{i}+\lambda_{i}^{*}\right)+\sum_{i=1}^{l} y_{i}\left(\lambda_{i}-\lambda_{i}^{*}\right)
$$

Subject to

$$
\sum\left(\lambda_{i}-\lambda_{i}^{*}\right)=0 \quad \lambda_{i}, \lambda_{i}^{*} \in(0, C)
$$

At the optimal solution, we obtain

$$
\begin{gathered}
w^{*}=\sum_{i=1}^{l}\left(\lambda_{i}-\lambda_{i}^{*}\right) x_{i} \\
f(x)=\sum_{i=1}^{l}\left(\lambda_{i}-\lambda_{i}^{*}\right) x_{i} x+b^{*}
\end{gathered}
$$


We compute the optimal value of $b$ from the complementary slackness conditions:

$$
\begin{array}{rlr}
b^{*}=y_{i}-w^{*} x_{i}-\varepsilon & \lambda_{i} \in(0, C) \\
\text { and } b^{*}=y_{i}-w^{*} x_{i}+\varepsilon & \lambda_{i}^{*} \in(0, C)
\end{array}
$$

In some case, we need to map input space into feature space and try to find a hyperplane in the feature space by using the trick of kernel functions:

$$
\operatorname{Max}-\frac{1}{2} \sum_{i=1}^{l} \sum_{j=1}^{l}\left(\lambda_{i}-\lambda_{i}^{*}\right)\left(\lambda_{j}-\lambda_{j}^{*}\right) K\left(x_{i}, x_{j}\right)-\varepsilon \sum_{i=1}^{l}\left(\lambda_{i}+\lambda_{i}^{*}\right)+\sum_{i=1}^{l} y_{i}\left(\lambda_{i}-\lambda_{i}^{*}\right)
$$

At the optimal solution, we obtain

$$
\begin{gathered}
w^{*}=\sum_{i=1}^{l}\left(\lambda_{i}-\lambda_{i}^{*}\right) K\left(x_{i}\right), \\
f(x)=\sum_{i=1}^{l}\left(\lambda_{i}-\lambda_{i}^{*}\right) K\left(x_{i}, x\right)+b
\end{gathered}
$$

where $\mathrm{K}(.,$.$) is a kernel function. Any symmetric positive semi-definite function,$ which satisfies Mercer's conditions, can be used as a kernel function in the SVMs context [10, 20]. In this paper, we use the linear kernel [11].

\section{Regressors for Comparison}

\subsection{Multivariate Linear Regression}

Multivariate Linear Regression (MLR) finds a set of basis vectors $w x_{i}$ and corresponding regressors $\beta_{i}$ in order to minimize the mean square error of the vector $y$. The basis vectors are described by the matrix $C x x^{-1} C x y$. A low-rank approximation to this problem can be defined by minimizing

$$
\varepsilon^{2}=E\left[\left\|y-\sum_{i=1}^{M} \beta_{i} w_{x_{i}}^{T} x w_{y_{i}}\right\|^{2}\right]
$$

where $M=\operatorname{dim}(y), N<M$ and the orthogonal basis $w y_{i}$ span the subspace of $y$ which gives the smallest mean square error given the rank $N$.

\subsection{Conjunctive Rule}

Conjunctive Rule (CR) consists of antecedents "AND"ed together and the consequent (prediction value) for the regression. This learner selects an antecedent by computing the Information Gain of each antecendent and prunes the generated rule using Reduced Error Prunning (REP) or simple pre-pruning based on the number of antecedents [1]. The Information is the weighted average of the mean-squared errors of both the data covered and not covered by the rule. In pruning, the weighted average of the mean-squared errors on the pruning data is used.

\subsection{Locally Weighted Regression}

Locally Weighted Regression (LWR) is a memory-based method that performs a regression around a point of interest using only training data that are local to that 
point $[3,4]$. We consider here a form of locally weighted regression that is a variant of the LOESS model [5]. The LOESS model performs a linear regression on points in the data set, weighted by a kernel centered at $x$. The kernel shape is a design parameter for which we use the Linear.

\section{Principal Component Analysis}

Principal Component Analysis (PCA) is a famous multivariate data analysis method that is useful in linear feature extraction [6,7]. The PCA finds a linear transformation $\mathrm{y}=\mathrm{Wx}$ such that the retained variance is maximized. Each row vector of $\mathrm{W}$ corresponds to the normalized orthogonal eigenvector of the data covariance matrix.

One simple approach to PCA is to use singular value decomposition (SVD). Let us denote the data covariance matrix by $\mathrm{R}_{\mathrm{x}}(0)=E\left\{\mathrm{x}(t) \mathrm{x}^{\mathrm{T}}(t)\right\}$. Then the SVD of $\mathrm{R}_{\mathrm{x}}(0)$ gives $R_{x}(0)=U D U^{T}$, where $U=\left[U_{s}, U_{n}\right]$ is the eigenvector matrix (i.e. modal matrix) and $\mathrm{D}$ is the diagonal matrix whose diagonal elements correspond to the eigenvalues of $\mathrm{R}_{\mathrm{x}}(0)$ (in descending order). Then the PCA transformation from $m$-dimensional data to $n$-dimensional subspace is given by choosing the first $n$ column vectors, i.e., $n$ principal component vector $\mathrm{y}$ is given by $\mathrm{y}=\mathrm{U}_{\mathrm{s}}^{\mathrm{T}} \mathrm{x}$.

\section{Experiment Results}

10-fold cross-validation on the benchmark MIS dataset, available on [9], is used for estimating prediction performance.

\subsection{Performance Measures}

We use the following performance measures:

Mean Absolute Error (MAE): MAE provides a measure of how close a prediction model is to the actual data.

$$
\text { MAE }=\frac{1}{n} \sum_{i=1}^{n}\left|a_{i}-p_{i}\right|
$$

where $a_{i}$ and $p_{i}$ is the actual and predicted value for the $i$ th test case. MAE ranges from 0 to infinity, with 0 corresponding to the ideal. the smaller the MAE the better.

Correlation Coefficient $(C C)$ : $\mathrm{CC}$ is a measure of how well trends in the predicted values follow trends in past actual values [21]. It measures how well the predicted values from a forecast model "fit" with the real-life data. A perfect fit gives a CC of 1.0 .

$$
\mathrm{CC}=\frac{\sum_{i=1}^{n}\left(p_{i}-\bar{p}\right)\left(a_{i}-\bar{a}\right)}{\sum_{i=1}^{n}\left(p_{i}-\bar{p}\right)^{2} \sum_{i=1}^{n}\left(a_{i}-\bar{a}\right)^{2}}
$$

The higher the $\mathrm{CC}$ the better.

\subsection{Results}

Fig.1 shows MAE of different regressors on the original MIS metrics and the PCA extracted data. On the original metrics, SVR with the linear kernel (SVR_L) get the 
best performance by achieving a minimum of 3.98 MAE. As shown in Fig.1, using the first few Principal Components (PC) we can still get relatively good performance. For CRule, using PCs is even better than using the original metrics.

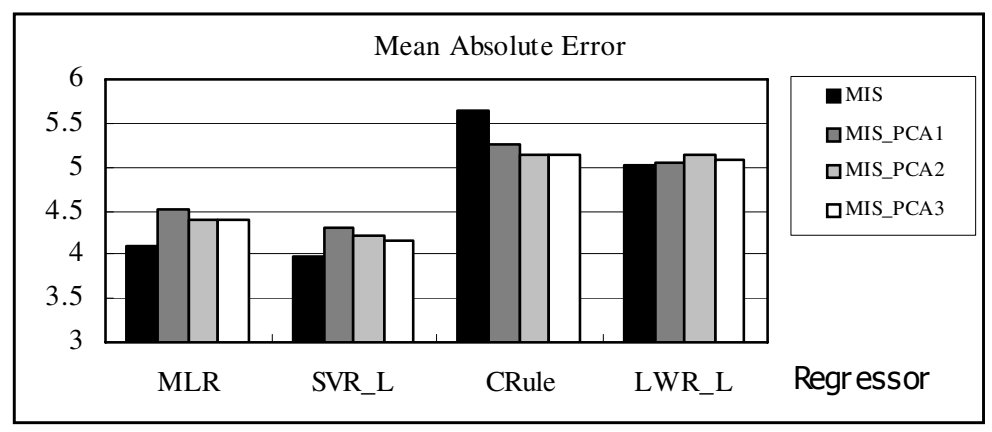

Fig. 1. MAE of different regressors on the original MIS metrics and the PCA extracted data. MLR= Multivariate Linear Regression, SVR_L=Support Vector Regression with Linear kernel, CRule=Conjunctive Rule, LWR_L=Locally Weighted Regression with Linear kernel. MIS_PCA1 means using the first Principal Component, MIS_PCA2 means using the first two Principal Components, etc.

Fig.2 shows CC of different regressors on the original MIS metrics and the PCA extracted data. SVR with Linear kernel get the best performance by achieving a maximum of 0.77 CC. For CRule, using PCs is better than using the original metrics.

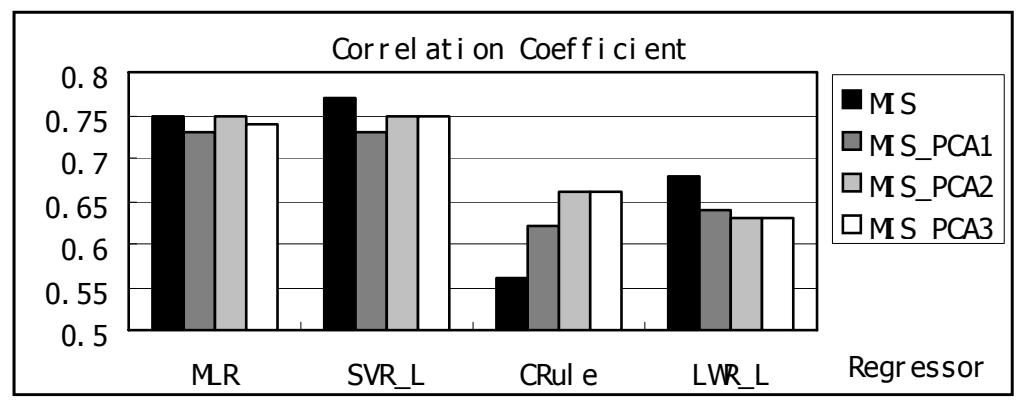

Fig. 2. CC of different regressors on the original MIS metrics and PCA extracted data. MLR= Multivariate Linear Regression, SVR_L=Support Vector Regression with Linear kernel, CRule=Conjunctive Rule, LWR_L=Locally Weighted Regression with Linear kernel. MIS_PCA1 means using the first Principal Component, MIS_PCA2 means using the first two Principal Components, etc.

\section{Conclusions}

In this paper we propose to use support vector machines for regression applied to software metrics to predict software quality. Comparison is done with three other 
techniques: Multivariate Linear Regression, Conjunctive Rule and Locally Weighted Regression. Results on benchmark dataset MIS, using MAE and CC as performance measures, indicate that support vector machines regression is a promising technique for software quality prediction. The investigation of PCA based metrics extraction show that using the first few Principal Components we can still get relatively good performance.

\section{Acknowledgments}

This work was supported by the National Science Foundation of China under the Grant No. 10001006 and No. 60273015.

\section{References}

1. I.Witten, E.Frank: Data Mining -Practical Machine Learning Tools and Techniques with Java Implementation. Morgan Kaufmann (2000)

2. Friedman, J.H.: Stochastic Gradient Boosting. Technical Report, Stanford University (1999)

3. Atkeson, C., A. Moore, S. Schaal: Locally Weighted Learning. AI Reviews (1996)

4. Cohn, D.A., Ghahramani, Z., Jordan, M.I.: Active Learning with Statistical Models. Journal of Artificial Intelligence Research, Vol. 4, pp. 129-145 (1996)

5. Cleveland, W., Devlin, S., Grosse, E.: Regression by Local Fitting. Journal of Econometrics, 37, pp. 87-114 (1988)

6. Zeng, X.Y., Chen, Y.W., et al: A New Texture Feature based on PCA Maps and Its Application to Image Retrieval. IEICE Trans. Inf. and Syst., E86-D 929-936 (2003)

7. Diamantaras, K.I. and Kung, S.Y.: Principal Component Neural Networks: Theory and Applications. John Wiley \& Sons, INC (1996)

8. D. Garmus, D. Herron: Measuring The Software Process, Prentice Hall, Upper Saddle River, NJ (1996)

9. MIS: http://www.win.tue.nl/ jromijn/2IW30/2IW30_statistics/LYU/DATA/CH12 (2006)

10. Theodore B. Trafalis, Huseyin Ince: Support Vector Machine for Regression and Applications to Financial Forecasting, ijcnn, p.6348, IEEE-INNS-ENNS International Joint Conference on Neural Networks (IJCNN'00) Volume 6 (2000)

11. A.J. Smola and B. Scholkopf, A Tutorial on Support Vector Regression, NEUROCOLT2 Technical Report Series, NC2-TR-1998-030 (1998)

12. Witold Pedrycz, Giancarlo Succi: Genetic Granular Classifiers in Modeling Software Quality. Journal of Systems and Software 76(3): 277-285 (2005)

13. W. Pedrycz, G. Succi, M.G. Chun, Association Analysis of Software Measures, Int. J of Software Engineering and Knowledge Engineering, 12(3): 291-316 (2002)

14. K.H. Muller, D.J. Paulish, Software Metrics, IEEE Press/Chapman \& Hall, London, 1993.

15. J. C. Munson, T. M. Khoshgoftaar: Software Metrics for Reliability Assessment, in Handbook of Software Reliability and System Reliability, McGraw-Hill, Hightstown, NJ, 1996.

16. 16.Scott Dick, Aleksandra Meeks, Mark Last, Horst Bunke, Abraham Kandel: Data Mining in Software Metrics Databases. Fuzzy Sets and Systems 145(1): 81-110 (2004)

17. W. Pedrycz, G. Succi, P. Musilek, X. Bai: Using Self-Organizing Maps to Analyze Object Oriented Software Measures. J. of Systems and Software, 59, 65-82 (2001) 
18. P. K. Simpson: Fuzzy Min-Max Neural Networks. Part 1: Classification, IEEE Trans. Neural Networks, Vol. 3, pp. 776-786 (1992)

19. M.S. Bazaraa, H.D. Sherali, and C.M. Shetty: Nonlinear Programming: Theory and Algorithms, John Wiley \&Sons Inc., New York (1993)

20. C. Cortes and V. Vapnik, Support Vector Networks, Machine Learning, 20, 273-297 (1995)

21. Correlation Coefficient: http://www.neatideas.com/cc.htm (2006)

22. T. Joachims, Making Large-Scale SVM Learning Practical, Technical Report, LS-8-24, Computer Science Department, University of Dortmund (1998)

23. R. Subramanyan and M.S. Krishnan: Empirical Analysis of CK Metrics for ObjectOriented Design Complexity: Implications for Software Defects, IEEE Trans. Software Eng., Vol. 29, pp. 297-310, Apr (2003) 